\title{
THE REPRESENTATIVE DOMAIN OF A HOMOGENEOUS BOUNDED DOMAIN
}

\author{
Hideyuki ISHI and Chifune KAI \\ (Received 16 March 2009)
}

\begin{abstract}
The representative domain gives a nice realization for a homogeneous bounded domain, which is a generalization of the Harish-Chandra realization for a symmetric bounded domain. We show that the representative domain coincides with the image of the Cayley transform introduced by Penney and Nomura. As an application, we see that a homogeneous bounded domain is symmetric if and only if its representative domain is convex.
\end{abstract}

\section{Introduction}

It is known that every homogeneous bounded domain is holomorphically equivalent to a homogeneous Siegel domain, which is a multi-dimensional generalization of the upper halfplane [20]. Moreover the Siegel domain realization is unique up to a linear transform [7]. Thus we may regard it as a canonical unbounded realization for a homogeneous bounded domain. On the other hand, a symmetric bounded domain has a canonical bounded realization called the Harish-Chandra realization, which is an open unit ball with respect to a certain norm on the ambient complex vector space. In addition, Korányi and Wolf [8] described the Cayley transform that maps the Harish-Chandra realization to the Siegel domain.

Then, it is natural to ask whether non-symmetric homogeneous bounded domains have nice bounded realizations. In 1996, Penney [17] modified a little the definition of the Harish-Chandra realization to obtain special bounded realizations for homogeneous bounded domains. Indeed, he introduced a specific biholomorphism, named the Cayley transform, which maps the associated Siegel domain to his bounded realization. When the domain is symmetric, his realization coincides with the Harish-Chandra realization and his Cayley transform is nothing but (the inverse of) the Cayley transform defined by Korányi and Wolf. Later, Nomura $[12,15]$ rediscovered Penney's Cayley transform through a computation in a study of harmonic analysis on homogeneous Siegel domains. Using the Cayley transform, Nomura $[13,14,16]$ proved some symmetry characterizations of homogeneous Siegel domains by geometric or analytic conditions. The second author of the present paper [4-6] discovered also the following relation between symmetry and convexity (partly a joint work with Nomura): a homogeneous Siegel domain is symmetric if and only if its Cayley transform image is convex. These facts imply that the Cayley transforms are actually significant.

2000 Mathematics Subject Classification: Primary 32H02; Secondary 32A25, 32M10, 32M15.

Keywords: Bergman kernel; representative domain; minimal domain; homogeneous bounded domain; symmetric bounded domain; Cayley transform.

(C) 2010 Faculty of Mathematics, Kyushu University 
However, it seems unclear how we characterize the Cayley transform image among bounded realizations for a homogeneous bounded domain.

On the other hand, in 1929, Bergman [1] had already tried to define canonical realizations for general bounded domains. His aim was to choose a 'representative' from each of the holomorphic equivalence classes of bounded domains. For a domain $D \subset \mathbb{C}^{N}$ holomorphically equivalent to a bounded domain and a fixed reference point $p \in D$, Bergman introduced a meromorphic mapping $\sigma_{p}^{D}: D \rightarrow \mathbb{C}^{N}$ by means of the Bergman kernel, and he called the image $\sigma_{p}^{D}(D)$ the representative domain of $D$. In this paper, we shall call $\sigma_{p}^{D}$ the Bergman mapping of $D$ defined at $p$ and write $R(D, p)$ instead of $\sigma_{p}^{D}(D)$. Lu [9] pointed out that the mapping $\sigma_{p}^{D}$ is not necessarily defined globally in general. If $\sigma_{p}^{D}$ is a global biholomorphism from $D$ onto $R(D, p)$, then the representative domain $R(D, p)$ has a number of nice properties. The most important one is its invariant nature under holomorphic isomorphisms (see Corollary 2.2).

Let $D$ be a homogeneous Siegel domain and $p$ any point in $D$. In this case, $\mathrm{Xu}$ [21] proved that the Bergman mapping $\sigma_{p}^{D}$ is a holomorphic isomorphism and $R(D, p)$ is a bounded domain. However, his proof is quite computational and not easy to follow. In the present paper, we show that the mapping $\sigma_{p}^{D}$ coincides with the Cayley transform defined by Penney and Nomura, so that the bijectivity of $\sigma_{p}^{D}$ and the boundedness of $R(D, p)$ follow also from their works. Besides, the coincidence enables us to give a simple definition of the Cayley transform, while the original definition is somewhat complicated. Furthermore, when a homogeneous bounded domain is given, the Cayley transform image of the associated Siegel domain can be obtained directly as the representative domain without considering the Siegel domain. As an application, we reformulate the second author's symmetry characterization theorem mentioned above in the following simple way: a homogeneous bounded domain is symmetric if and only if its representative domain is convex.

In addition, we can capture some properties of the Cayley transform image of a homogeneous Siegel domain, which is now equal to the representative domain, by applying general results in $[\mathbf{1}, \mathbf{1 0}, \mathbf{1 1}]$.

(i) The isotropy subgroup of the representative domain at the origin consists of unitary linear mappings.

(ii) The representative domain is characterized by means of second derivatives of the Bergman kernel up to an affine transform.

(iii) The representative domain is a minimal domain, that is, its Euclidean volume is minimal in a certain sense.

Perhaps in future studies, these facts might afford a clue to a true characterization of the Cayley transform image among bounded realizations.

The present paper is organized as follows. In Sections 2 and 3, we summarize fundamental facts concerning the Bergman mappings and the representative domains used in this paper. These results are not new. Though most of them can be verified easily, we include the proofs for the reader's convenience except for a few propositions. In Section 2, after a review of basic definitions, we present the unitarization property of the Bergman mappings. In Section 2.1, we go further under a strong condition that the Bergman mapping is a global biholomorphism on the given domain. In Section 2.2, it is verified that the Bergman mapping of a bounded circular domain defined at the origin becomes a positive definite Hermitian linear transform. Then an alternative proof of the uniqueness theorem of Cartan is also 
presented. Section 3 is concerned with homogeneous bounded domains. Here it is proved that the representative domain of a symmetric bounded domain coincides essentially with the Harish-Chandra realization by using the results of Section 2.2. In Section 3.1, we see that the representative domain of a homogeneous bounded domain is a minimal domain in the sense of Bergman. Section 4 contains our main result. Generalizing the definition by Penney and Nomura naturally, we introduce the Cayley transform for a general (not necessarily homogeneous) Siegel domain, and show that our Cayley transform coincides with 1/2 multiple of the Bergman mapping. In particular, since Penney's Cayley transform for a homogeneous Siegel domain turns out to be the Bergman mapping, we can interpret the results in $[\mathbf{4 - 6 , 1 2 - 1 4 , 1 7 ] ~ i n ~ t h e ~ c o n t e x t ~ o f ~ t h e ~ s t u d y ~ o f ~ t h e ~ r e p r e s e n t a t i v e ~ d o m a i n ~ o f ~ a ~}$ homogeneous bounded domain. One such application is the second author's characterization theorem reformulated in Section 5.

\section{Bergman mappings and representative domains}

Let $D \subset \mathbb{C}^{N}$ be a domain biholomorphic to a bounded domain, and $H^{2}(D)$ the Bergman space on $D$, that is, the Hilbert space consisting of square integrable holomorphic functions on $D$. Taking an orthonormal basis $\left\{\phi_{\alpha}\right\}_{\alpha \in \mathbb{N}}$ of $H^{2}(D)$, we define the Bergman kernel $K_{D}: D \times D \rightarrow \mathbb{C}$ by

$$
K_{D}(z, \zeta):=\sum_{\alpha \in \mathbb{N}} \phi_{\alpha}(z) \overline{\phi_{\alpha}(\zeta)} \quad(z, \zeta \in D)
$$

which is independent of the choice of $\left\{\phi_{\alpha}\right\}_{\alpha \in \mathbb{N}}$. Note that, since $D$ is biholomorphic to a bounded domain, we have $K_{D}(z, z)>0(z \in D)$. For $z, \zeta \in D$ with $K_{D}(z, \zeta) \neq 0$, we define an $N \times N$ Hermitian matrix $T_{D}(z, \zeta)$ by

$$
T_{D}(z, \zeta):=\left(\begin{array}{ccc}
\frac{\partial^{2}}{\partial \bar{\zeta}_{1} \partial z_{1}} \log K_{D}(z, \zeta) & \cdots & \frac{\partial^{2}}{\partial \bar{\zeta}_{1} \partial z_{N}} \log K_{D}(z, \zeta) \\
\vdots & \ddots & \vdots \\
\frac{\partial^{2}}{\partial \bar{\zeta}_{N} \partial z_{1}} \log K_{D}(z, \zeta) & \cdots & \frac{\partial^{2}}{\partial \bar{\zeta}_{N} \partial z_{N}} \log K_{D}(z, \zeta)
\end{array}\right)
$$

For every $z \in D$, the Hermitian matrix $T_{D}(z, z)$ is positive definite [2], which represents the so-called Bergman metric at $z$. In what follows, we write $T_{D}(z)$ instead of $T_{D}(z, z)$ for simplicity. Let $\varphi: D \stackrel{\sim}{\rightarrow} D^{\prime}$ be a biholomorphism onto a domain $D^{\prime}$. For $z \in D$, we denote by $J(\varphi, z)$ the Jacobian matrix of $\varphi={ }^{\mathrm{t}}\left(\varphi_{1}, \ldots, \varphi_{N}\right)$ at $z$ :

$$
J(\varphi, z)=\left(\begin{array}{ccc}
\frac{\partial \varphi_{1}}{\partial z_{1}}(z) & \cdots & \frac{\partial \varphi_{1}}{\partial z_{N}}(z) \\
\vdots & \ddots & \vdots \\
\frac{\partial \varphi_{N}}{\partial z_{1}}(z) & \cdots & \frac{\partial \varphi_{N}}{\partial z_{N}}(z)
\end{array}\right)
$$

For $z, \zeta \in D$ we have

$$
K_{D}(z, \zeta)=\overline{\operatorname{det} J(\varphi, \zeta)} K_{D^{\prime}}(\varphi(z), \varphi(\zeta)) \operatorname{det} J(\varphi, z)
$$


and, if $K_{D}(z, \zeta) \neq 0$, we also have

$$
T_{D}(z, \zeta)=\bar{J}(\varphi, \zeta) T_{D^{\prime}}(\varphi(z), \varphi(\zeta)) J(\varphi, z)
$$

For anti-holomorphic functions $f(\zeta)$ on $D$, we set

$$
\operatorname{grad}_{\bar{\zeta}} f(\zeta):={ }^{\mathrm{t}}\left(\frac{\partial f}{\partial \bar{\zeta}_{1}}(\zeta), \ldots, \frac{\partial f}{\partial \bar{\zeta}_{N}}(\zeta)\right)
$$

Let us fix a point $p \in D$. Setting

$$
U_{p}^{D}:=\left\{z \in D \mid K_{D}(z, p) \neq 0\right\},
$$

we define a mapping $\sigma_{p}^{D}: U_{p}^{D} \rightarrow \mathbb{C}^{N}$ by

$$
\sigma_{p}^{D}(z):=\left.T_{D}(p)^{-1 / 2} \operatorname{grad}_{\zeta} \log \frac{K_{D}(z, \zeta)}{K_{D}(p, \zeta)}\right|_{\zeta=p} \quad\left(z \in U_{p}^{D}\right),
$$

where $T_{D}(p)^{1 / 2}$ stands for the square root of the positive definite Hermitian matrix $T_{D}(p)$. Since $K_{D}(p, p) \neq 0$, the mapping $\sigma_{p}^{D}$ is well defined. We call $\sigma_{p}^{D}$ the Bergman mapping defined at $p$. Clearly, $\sigma_{p}^{D}$ is holomorphic. Since

$$
J\left(\sigma_{p}^{D}, z\right)=T_{D}(p)^{-1 / 2} J\left(\left.\operatorname{grad}_{\zeta} \log \frac{K_{D}(z, \zeta)}{K_{D}(p, \zeta)}\right|_{\zeta=p}, z\right) \quad\left(z \in U_{p}^{D}\right)
$$

and

$$
\frac{\partial}{\partial z_{k}}\left(\left.\frac{\partial}{\partial \bar{\zeta}_{j}} \log \frac{K_{D}(z, \zeta)}{K_{D}(p, \zeta)}\right|_{\zeta=p}\right)=\left.\frac{\partial^{2}}{\partial \bar{\zeta}_{j} \partial z_{k}} \log K_{D}(z, \zeta)\right|_{\zeta=p} \quad\left(z \in U_{p}^{D}, 1 \leq j, k \leq N\right)
$$

we obtain

$$
J\left(\sigma_{p}^{D}, z\right)=T_{D}(p)^{-1 / 2} T_{D}(z, p) \quad\left(z \in U_{p}^{D}\right) .
$$

In particular, we have

$$
J\left(\sigma_{p}^{D}, p\right)=T_{D}(p)^{1 / 2} .
$$

Hence we can take a neighborhood $V_{p} \subset U_{p}^{D}$ of $p$ in such a way that $\left.\sigma_{p}^{D}\right|_{V_{p}}$ gives a local coordinate around $p$ with

$$
\sigma_{p}^{D}(p)=0 .
$$

If $K_{D}(z, p) \neq 0$ for all $z \in D$, then $\sigma_{p}^{D}$ is defined on $D=U_{p}^{D}$. In this case, we set $R(D, p):=$ $\sigma_{p}^{D}(D)$, which we call the representative domain of $D$ along the lines of Bergman, though $R(D, p)$ is not necessarily a domain in general.

Let $\varphi: D \stackrel{\sim}{\rightarrow} D^{\prime}$ be a biholomorphism. We define an $N \times N$ matrix $L(\varphi, p)$ by

$$
L(\varphi, p):=T_{D^{\prime}}(\varphi(p))^{-1 / 2} \overline{\mathrm{t}}^{J(\varphi, p)}-1 T_{D}(p)^{1 / 2} .
$$

By (2.2), $L(\varphi, p)$ is a unitary matrix. We also denote by the same symbol $L(\varphi, p)$ the linear mapping $\mathbb{C}^{N} \ni z \mapsto L(\varphi, p) z \in \mathbb{C}^{N}$. Note that, for $z \in D$ with $K_{D}(z, p) \neq 0$, we have $K_{D^{\prime}}(\varphi(z), \varphi(p)) \neq 0$ by $(2.1)$, so that $\varphi\left(U_{p}^{D}\right)=U_{\varphi(p)}^{D^{\prime}}$. Thus $\sigma_{\varphi(p)}^{D^{\prime}} \circ \varphi$ is well defined. The following unitarization of $\varphi$ is a distinctive feature of the Bergman mapping. 
Proposition 2.1. One has $\sigma_{\varphi(p)}^{D^{\prime}} \circ \varphi=L(\varphi, p) \circ \sigma_{p}^{D}$. In other words, the following diagram is commutative.

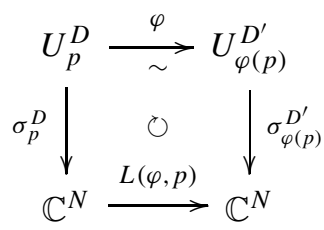

Proof. For $z \in U_{p}^{D}$, we have by (2.1)

$$
\frac{K_{D}(z, \zeta)}{K_{D}(p, \zeta)}=\frac{K_{D^{\prime}}(\varphi(z), \varphi(\zeta))}{K_{D^{\prime}}(\varphi(p), \varphi(\zeta))} \frac{\operatorname{det} J(\varphi, z)}{\operatorname{det} J(\varphi, p)}
$$

whence it follows that

$$
\left.\operatorname{grad}_{\bar{\zeta}} \log \frac{K_{D}(z, \zeta)}{K_{D}(p, \zeta)}\right|_{\zeta=p}=\left.\operatorname{grad}_{\bar{\zeta}} \log \frac{K_{D^{\prime}}(\varphi(z), \varphi(\zeta))}{K_{D^{\prime}}(\varphi(p), \varphi(\zeta))}\right|_{\zeta=p} .
$$

By the change of variable $\xi:=\varphi(\zeta)$, the right-hand side is rewritten as

$$
\left.\mathrm{t} \overline{J(\varphi, p)} \operatorname{grad}_{\bar{\xi}} \log \frac{K_{D^{\prime}}(\varphi(z), \xi)}{K_{D^{\prime}}(\varphi(p), \xi)}\right|_{\xi=\varphi(p)} .
$$

This together with (2.4) and (2.8) gives

$$
\begin{aligned}
L(\varphi, p) \sigma_{p}^{D}(z) & =\left.L(\varphi, p) T_{D}(p)^{-1 / 2} \mathrm{t} \frac{}{J(\varphi, p)} \operatorname{grad}_{\bar{\xi}} \log \frac{K_{D^{\prime}}(\varphi(z), \xi)}{K_{D^{\prime}}(\varphi(p), \xi)}\right|_{\xi=\varphi(p)} \\
& =\sigma_{\varphi(p)}^{D^{\prime}}(\varphi(z)) .
\end{aligned}
$$

Corollary 2.2. If $K_{D}(z, p) \neq 0$ for all $z \in D$, then $R\left(D^{\prime}, \varphi(p)\right)=L(\varphi, p) R(D, p)$.

Namely, the representative domain up to a unitary transform gives an invariant of the pair $(D, p)$ with respect to biholomorphisms.

\subsection{The case that $\sigma_{p}^{D}$ is a global biholomorphism}

In this subsection, we assume that $K_{D}(z, p) \neq 0$ for all $z \in D$ and, in addition, that $\sigma_{p}^{D}$ gives a biholomorphism from $D$ onto $R(D, p)$. Note that $K_{R(D, p)}(w, 0) \neq 0(w \in R(D, p))$ by (2.1) and (2.7). Hence, $\sigma_{0}^{R(D, p)}$ gives a holomorphic mapping $R(D, p) \rightarrow \mathbb{C}^{N}$. We denote by $E_{N}$ the $N \times N$ unit matrix.

\section{PROPOSITION 2.3.}

(1) For $w \in R(D, p)$, one has $T_{R(D, p)}(w, 0) \equiv E_{N}$.

(2) $\sigma_{0}^{R(D, p)}$ is the identity mapping.

Proof. (1) We take $z \in D$ such that $\sigma_{p}^{D}(z)=w$. In (2.2), we set $\varphi:=\sigma_{p}^{D}, D^{\prime}:=R(D, p)$ and $\zeta:=p$. Then by (2.5), (2.6) and (2.7), we obtain that

$$
T_{D}(z, p)=T_{D}(p)^{1 / 2} T_{R(D, p)}(w, 0) T_{D}(p)^{-1 / 2} T_{D}(z, p) \quad(z \in D) .
$$

Since $\sigma_{p}^{D}$ is a biholomorphism, $T_{D}(z, p)(z \in D)$ is regular by (2.5). Therefore we have $E_{N}=T_{D}(p)^{1 / 2} T_{R(D, p)}(w, 0) T_{D}(p)^{-1 / 2}$, which implies that $T_{R(D, p)}(w, 0)=E_{N}$. 
(2) Setting $\varphi:=\sigma_{p}^{D}$ in Proposition 2.1, we see by (2.7) that $\sigma_{0}^{R(D, p)} \circ \sigma_{p}^{D}=L\left(\sigma_{p}^{D}, p\right) \circ$ $\sigma_{p}^{D}$. Since $\sigma_{p}^{D}$ is a biholomorphism, it follows that $\sigma_{0}^{R(D, p)}=L\left(\sigma_{p}^{D}, p\right)$. By the assertion (1), we obtain $T_{R(D, p)}(0)=E_{N}$. This together with (2.8) and (2.6) gives $L\left(\sigma_{p}^{D}, p\right)=E_{N}$, which completes the proof.

COROLlARY 2.4. If $g \in \operatorname{Hol}(R(D, p))$ and $g(0)=0$, then $g$ is a unitary linear mapping.

Proof. In Proposition 2.1, we set $D=D^{\prime}:=R(D, p), \varphi:=g$ and $p:=0$. Then we have $\sigma_{0}^{R(D, p)}(g(z))=L(g, 0) \sigma_{0}^{R(D, p)}(z)$ for $z \in R(D, p)$. This together with Proposition 2.3(2) completes the proof.

\subsection{The representative domain of a circular bounded domain}

Let $\mathcal{D} \subset \mathbb{C}^{N}$ be a circular bounded domain with $0 \in \mathcal{D}$.

\section{LEMMA 2.5 .}

(1) $K_{\mathcal{D}}(z, 0)(z \in \mathcal{D})$ is a non-zero constant.

(2) $T_{\mathcal{D}}(z, 0)(z \in \mathcal{D})$ is a constant matrix, so that it equals the positive definite Hermitian matrix $T_{\mathcal{D}}(0)$.

Proof. (1) Let $\theta \in \mathbb{R}$ and $\varphi_{\theta}(z):=e^{i \theta} z(z \in \mathcal{D})$. In (2.1), we set $D=D^{\prime}:=\mathcal{D}, \varphi:=\varphi_{\theta}$ and $\zeta:=0$. Then we obtain

$$
K_{\mathcal{D}}(z, 0)=K_{\mathcal{D}}\left(e^{i \theta} z, 0\right) \quad(z \in \mathcal{D})
$$

Considering the Taylor expansion of $K_{\mathcal{D}}(z, 0)$ at $z=0$, we see that $K_{\mathcal{D}}(z, 0)(z \in \mathcal{D})$ is constant. It is non-zero, because $K_{\mathcal{D}}(0,0)>0$.

(2) By a similar argument using (2.2), the assertion (2) follows. of $\mathcal{D}$.

Thanks to Lemma 2.5, we now have the holomorphic mapping $\sigma_{0}^{\mathcal{D}}$ defined on the whole

\section{PROPOSITION 2.6.}

(1) $\sigma_{0}^{\mathcal{D}}(z)=T_{\mathcal{D}}(0)^{1 / 2} z(z \in \mathcal{D})$. In particular, one has $R(\mathcal{D}, 0)=T_{\mathcal{D}}(0)^{1 / 2} \mathcal{D}$.

(2) $[$ Cartan $]$ If $g \in \operatorname{Hol}(\mathcal{D})$ and $g(0)=0$, then $g$ is linear.

Proof. (1) Since $T_{\mathcal{D}}(z, 0)$ is constant for $z \in \mathcal{D}$, it follows from (2.5) that the Jacobian matrix $J\left(\sigma_{0}^{\mathcal{D}}, z\right)$ equals $T_{\mathcal{D}}(0)^{-1 / 2} T_{\mathcal{D}}(z, 0)=T_{\mathcal{D}}(0)^{1 / 2}$, which is also constant. Thus $\sigma_{0}^{\mathcal{D}}$ is an affine transform, while $\sigma_{0}^{\mathcal{D}}(0)=0$ by (2.7). Hence $\sigma_{0}^{\mathcal{D}}(z)=T_{\mathcal{D}}(0)^{1 / 2} z$.

(2) Setting $D=D^{\prime}:=\mathcal{D}$ and $\varphi:=g$ in Proposition 2.1, we see that

$$
\sigma_{0}^{\mathcal{D}}(g(z))=L(g, 0) \sigma_{0}^{\mathcal{D}}(z) \quad(z \in \mathcal{D})
$$

This together with (1) gives

$$
g(z)=T_{\mathcal{D}}(0)^{-1 / 2} L(g, 0) T_{\mathcal{D}}(0)^{1 / 2} z
$$




\section{The representative domain of a homogeneous bounded domain}

Throughout this section, we suppose that $D \subset \mathbb{C}^{N}$ is a domain biholomorphic to a homogeneous bounded domain. Then $D$ is biholomorphic to a homogeneous Siegel domain, whose Bergman kernel was described explicitly and turned out to be zero-free in [3] (see also Section 4.1 of the present paper). Hence by (2.1) we know the following.

Proposition 3.1. For all $z, \zeta \in D$, one has $K_{D}(z, \zeta) \neq 0$.

Thus, for any $p \in D$, the Bergman mapping $\sigma_{p}^{D}$ gives a global holomorphic mapping on $D$. We note that the choice of the reference point $p$ is not essential in the following sense.

LemmA 3.2. For $p_{1}, p_{2} \in D$, the representative domain $R\left(D, p_{2}\right)$ coincides with $R\left(D, p_{1}\right)$ up to a unitary transform.

Proof. By the homogeneity of $D$, there exists $g \in \operatorname{Hol}(D)$ such that $g\left(p_{1}\right)=p_{2}$. Hence we have $R\left(D, p_{2}\right)=L\left(g, p_{1}\right) R\left(D, p_{1}\right)$ by Corollary 2.2 .

The following theorem, which was first proved by $\mathrm{Xu}$ [21] quite computationally, is easily deduced from Theorem 4.1 and [17] (or [12]), whereas we state it here for the convenience of the argument.

THEOREM 3.3. For any fixed $p \in D$, the Bergman mapping $\sigma_{p}^{D}$ gives a biholomorphism from $D$ onto the representative domain $R(D, p)$. Moreover, $R(D, p)$ is bounded.

The following proposition gives a characterization of the representative domain $R(D, p)$ among bounded realizations for the homogeneous bounded domain $D$ (cf. [10]).

Proposition 3.4. Let $\mathcal{D}$ be a bounded domain biholomorphic to D. Assume that there exists a point $t \in \mathcal{D}$ for which $T_{\mathcal{D}}(z, t)$ is a constant matrix for $z \in \mathcal{D}$. Then $\mathcal{D}$ is an affine transform of the representative domain $R(D, p)$.

Proof. Since $R(D, p)$ and $\mathcal{D}$ are biholomorphic to $D$, which is homogeneous, there exists a biholomorphism $\psi: R(D, p) \simeq \mathcal{D}$ with $\psi(0)=t$. It follows from (2.2) that

$$
T_{R(D, p)}(z, 0)={ }^{\mathrm{t}} \overline{J(\psi, 0)} T_{\mathcal{D}}(\psi(z), t) J(\psi, z) \quad(z \in R(D, p)) .
$$

This together with Proposition 2.3(1) and the assumption on $\mathcal{D}$ tells us that $J(\psi, z)$ is a constant matrix for $z \in R(D, p)$. Thus, $\psi$ is extended to a non-degenerate affine transform on $\mathbb{C}^{N}$.

In the special case that $D$ is a symmetric bounded domain, it is known that $D$ has a canonical bounded realization called the Harish-Chandra realization. Namely, $D$ is realized as an open unit ball with respect to a certain norm, so that it is convex and circular with the origin inside [19, Ch. II, Section 4]. Since the Harish-Chandra realization is circular, from Lemma 2.5(2) and Proposition 3.4 we obtain the following result.

PROPOSITION 3.5. If $D$ is a symmetric bounded domain, then $R(D, p)$ is a linear transform of the Harish-Chandra realization for $D$. 


\subsection{Minimality of the volume of the representative domain}

Let $\mathcal{D} \subset \mathbb{C}^{N}$ be a finite-volume domain and $t \in \mathcal{D}$. We say that $\mathcal{D}$ is a minimal domain with a center $t$ if the following condition is satisfied: for every biholomorphism $\psi: \mathcal{D} \simeq \mathcal{D}^{\prime}$ with $\operatorname{det} J(\psi, t)=1$, we have

$$
\operatorname{vol}\left(\mathcal{D}^{\prime}\right) \geq \operatorname{vol}(\mathcal{D})
$$

where $\operatorname{vol}(\mathcal{D})$ stands for the Euclidean volume of $\mathcal{D}$.

Proposition 3.6. [11, Theorem 3.1] Let $\mathcal{D} \subset \mathbb{C}^{N}$ be a bounded univalent domain and $t \in \mathcal{D}$. Then, $\mathcal{D}$ is a minimal domain with a center $t$ if and only if $K_{\mathcal{D}}(z, t)(z \in \mathcal{D})$ is constant on $\mathcal{D}$.

In general, the representative domain is not necessarily minimal (see [11]). Nevertheless, it is the case for a homogeneous bounded domain. First we show the following lemma.

LEMMA 3.7. If $\mathcal{D} \subset \mathbb{C}^{N}$ is a domain biholomorphic to a homogeneous bounded domain, the ratio

$$
\frac{K_{\mathcal{D}}(z, \zeta)}{\operatorname{det} T_{\mathcal{D}}(z, \zeta)} \quad(z, \zeta \in \mathcal{D})
$$

is constant on $\mathcal{D} \times \mathcal{D}$.

Proof. Since the ratio is holomorphic in $z$ and anti-holomorphic in $\zeta$, it is enough to show that the ratio is constant on $\{(z, z) \mid z \in \mathcal{D}\}$. Let us fix any $z \in \mathcal{D}$ and take any $\xi \in \mathcal{D}$. Since $\mathcal{D}$ is homogeneous, there exists $\varphi \in \operatorname{Hol}(\mathcal{D})$ such that $\varphi(z)=\xi$. Then we see from (2.1) and (2.2) that

$$
\frac{K_{\mathcal{D}}(z, z)}{\operatorname{det} T_{\mathcal{D}}(z, z)}=\frac{K_{\mathcal{D}}(\xi, \xi)}{\operatorname{det} T_{\mathcal{D}}(\xi, \xi)}
$$

which completes the proof.

PROPOSITION 3.8. The representative domain $R(D, p)$ is a minimal domain with a center 0.

Proof. It follows from Proposition 2.3(1) that det $T_{R(D, p)}(z, 0) \equiv 1(z \in R(D, p))$. Hence we know by Lemma 3.7 that $K_{R(D, p)}(z, 0)$ is constant for $z \in R(D, p)$. This together with Proposition 3.6 tells us that $R(D, p)$ is a minimal domain with a center 0 .

\section{The Cayley transform for a Siegel domain}

First we recall the definition of Siegel domains. Let $\Omega$ be an open convex cone in a finitedimensional real vector space $V$. We suppose that the cone $\Omega$ is regular, that is, $\Omega$ contains no straight line. We set $W:=V_{\mathbb{C}}$, the complexification of $V$ and denote by $w \mapsto w^{*}$ the conjugation of $W$ relative to the real form $V$. Let $U$ be a finite-dimensional complex vector space and $Q: U \times U \rightarrow W$ a sesquilinear Hermitian mapping. We suppose that $Q: U \times U \rightarrow W$ is $\Omega$-positive, that is, one has $Q(u, u) \in \mathrm{Cl}(\Omega) \backslash\{0\}$ for all $u \in U \backslash\{0\}$, where $\mathrm{Cl}(\Omega)$ stands for the closure of $\Omega$. The Siegel domain $\mathscr{D}$ corresponding to these data is defined by

$$
\mathscr{D}:=\left\{(u, w) \in U \times W \mid \operatorname{Re} w-\frac{1}{2} Q(u, u) \in \Omega\right\} .
$$


In what follows, for functions $f$ on a complex vector space $Z$ and $z, z^{\prime} \in Z, D_{z} f\left(z^{\prime}\right)$ stands for the directional derivative in the direction $z$ at $z^{\prime}$ :

$$
D_{z} f\left(z^{\prime}\right):=\left.\frac{d}{d t} f\left(z^{\prime}+t z\right)\right|_{t=0} .
$$

The holomorphic and anti-holomorphic derivatives are denoted by $\partial_{z} f:=\left(D_{z} f-i D_{i z} f\right) / 2$ and $\bar{\partial}_{z} f:=\left(D_{z} f+i D_{i z} f\right) / 2$ respectively.

\subsection{The Cayley transform associated with the Bergman kernel}

Since $\mathscr{D}$ is biholomorphic to a bounded domain [18], $\mathscr{D}$ has the Bergman kernel $K_{\mathscr{D}}: \mathscr{D} \times$ $\mathscr{D} \rightarrow \mathbb{C}$. Inspired by Penney's [17] and Nomura's [12] works, we shall define the Cayley transform for $\mathscr{D}$ associated with $K_{\mathscr{D}}$. By [3, Proposition 5.1], there exists a holomorphic function $\eta$ on $\Omega+i V$ for which $K_{\mathscr{D}}$ is expressed in the following form:

$$
K_{\mathscr{D}}\left(z_{1}, z_{2}\right)=\eta\left(w_{1}+w_{2}^{*}-Q\left(u_{1}, u_{2}\right)\right) \quad\left(z_{j}=\left(u_{j}, w_{j}\right) \in \mathscr{D}, j=1,2\right) .
$$

We define a meromorphic mapping $\mathcal{I}: \Omega+i V \rightarrow W^{*}$ by

$$
\left\langle w, \mathcal{I}\left(w^{\prime}\right)\right\rangle=-\partial_{w} \log \eta\left(w^{\prime}\right) \quad\left(w \in W, w^{\prime} \in \Omega+i V\right),
$$

which we call the pseudo-inverse mapping. For $x \in W$, we denote by $\mathrm{x}$ the point $(0, x) \in$ $U \times W$. If $x \in \Omega$, then $\mathbf{x} / 2 \in \mathscr{D}$ by (4.1). Since $K_{\mathscr{D}}(\mathbf{x} / 2, \mathbf{x} / 2)>0$ and $K_{\mathscr{D}}(\mathbf{x} / 2, \mathbf{x} / 2)=\eta(x)$ by (4.2), we see that $\eta$ is positive on $\Omega$. Hence $\mathcal{I}$ is defined at every $x \in \Omega$. By [3], $\eta$ is homogeneous, that is, there exists a negative integer $k$ for which

$$
\eta(\lambda w)=\lambda^{k} \eta(w) \quad(\lambda>0, w \in \Omega+i V),
$$

whence it follows easily that

$$
\mathcal{I}(\lambda w)=\lambda^{-1} \mathcal{I}(w) \quad(\lambda>0, w \in \Omega+i V) .
$$

We fix any point $E \in \Omega$, and set $\mathrm{e}:=(0, E) \in \mathscr{D}$. Recalling (2.3), we observe that the set $U_{\mathrm{e}}^{\mathscr{D}}=\left\{z \in \mathscr{D} \mid K_{\mathscr{D}}(z, \mathrm{e}) \neq 0\right\}$ is described as

$$
U_{\mathrm{e}}^{\mathscr{D}}=\{(u, w) \in \mathscr{D} \mid \eta(w+E) \neq 0\}
$$

owing to (4.2). Let $U^{\dagger}$ be the space of all antilinear forms on $U$. Noting that $\mathcal{I}(E) \in W^{*}$ is well defined, we define a meromorphic mapping $\mathcal{C}=\mathcal{C}_{\mathrm{e}}: \mathscr{D} \rightarrow U^{\dagger} \times W^{*}$ called the Cayley transform for $\mathscr{D}$ by

$$
\mathcal{C}(u, w):=(\langle 2 Q(u, \cdot), \mathcal{I}(w+E)\rangle, \mathcal{I}(E)-2 \mathcal{I}(w+E)) \quad((u, w) \in \mathscr{D}) .
$$

Thanks to (4.5), $\mathcal{C}$ is defined on $U_{\mathrm{e}}^{\mathscr{D}}$.

Here we make a few remarks on the case that $\mathscr{D}$ is homogeneous. In this case, we know by [18] that $\Omega$ is a homogeneous convex cone, that is, the linear automorphism group $G(\Omega)$ of $\Omega$ defined by

$$
G(\Omega):=\{g \in G L(V) \mid g(\Omega)=\Omega\}
$$

acts transitively on $\Omega$. It follows from [3, Lemma 5.1] and the note right after [3, Proposition 2.3] that $\eta$ is zero-free on $\Omega+i V$. Hence, by (4.3), $\mathcal{I}: \Omega+i V \rightarrow W^{*}$ is a 
holomorphic mapping. Moreover, we see from [12, Proposition 2.6] and [12, Theorem 2.11] that $\mathcal{I}$ is a biholomorphic mapping from $\Omega+i V$ onto $\mathcal{I}(\Omega+i V)$. Furthermore, if the reference point $\mathrm{e}$ is specified as in [12], we know by [12, Theorem 3.6] that $\mathcal{C}=\mathcal{C}_{\mathrm{e}}$ is a biholomorphic mapping from $\mathscr{D}$ onto $\mathcal{C}(\mathscr{D})$ and by [12, Lemma 3.1] that $\mathcal{C}(\mathscr{D})$ is bounded.

We note that it is an open question whether $\eta$ for a non-homogeneous Siegel domain is zero-free on $\Omega+i V$. Even if $\mathcal{C}$ is defined globally on $\mathscr{D}$, it is not known either whether the image $\mathcal{C}(\mathscr{D})$ is bounded.

\subsection{Coincidence of the Bergman mapping and the Cayley transform}

We keep to the notation in Section 4.1. In order to compare our Cayley transform $\mathcal{C}=$ $\mathcal{C}_{\mathrm{e}}: \mathscr{D} \rightarrow U^{\dagger} \times W^{*}$ and the Bergman mapping $\sigma_{\mathrm{e}}^{\mathscr{D}}: \mathscr{D} \rightarrow \mathbb{C}^{N}(N:=\operatorname{dim} \mathscr{D})$, we shall identify the vector space $U^{\dagger} \times W^{*}$ with $\mathbb{C}^{N}$ in the following way. Let $\theta: U \times W \rightarrow U \times W$ be the mapping given by $\theta(u, w):=\left(u, w^{*}\right)$. Then, if $f \in U^{\dagger} \times W^{*}$, we have $f \circ \theta \in$ $(U \times W)^{\dagger}$. Let us introduce a Hermitian sesquilinear form on $U \times W$ by

$$
\left(z_{1} \mid z_{2}\right):=\left.\partial_{z_{1}} \bar{\partial}_{z_{2}} \log K_{\mathscr{D}}(z, z)\right|_{z=\mathrm{e}} \quad\left(z_{1}, z_{2} \in U \times W\right) .
$$

This defines a positive definite Hermitian inner product, which is nothing but the Bergman metric at e. For $f \in U^{\dagger} \times W^{*}$, we define $\hat{f} \in U \times W$ by

$$
(\hat{f} \mid z)=\langle z, f \circ \theta\rangle \quad(z \in U \times W) .
$$

On the other hand, we take any orthonormal basis of $U \times W$ with respect to $(\cdot \mid \cdot)$, and identify $\mathbb{C}^{N}$ with $U \times W$ by means of the basis. Note that $T_{\mathscr{D}}(\mathrm{e})=E_{N}$ in the notation of Section 2.

THEOREM 4.1. Under the identification $U^{\dagger} \times W^{*} \ni f \mapsto \hat{f} \in \mathbb{C}^{N}$, for $z \in U_{e}^{\mathscr{D}}$ one has

$$
\sigma_{e}^{\mathscr{D}}(z)=\frac{1}{2} \mathcal{C}(z)^{\wedge}
$$

In particular, if $U_{e}^{\mathscr{D}}=\mathscr{D}$, one has

$$
R(\mathscr{D}, e)=\frac{1}{2} \mathcal{C}(\mathscr{D}) \hat{.}
$$

Proof. For $z_{j}=\left(u_{j}, w_{j}\right) \in \mathscr{D}\left(j=1,2, u_{j} \in U, w_{j} \in W\right)$, we set

$$
\Phi\left(z_{1}, z_{2}\right):=w_{1}+w_{2}^{*}-Q\left(u_{1}, u_{2}\right) \in \Omega+i V .
$$

We fix any point $z=(u, w) \in U_{\mathrm{e}}^{\mathscr{D}}$. Then, $\log K_{\mathscr{D}}(z, \zeta)$ is an anti-holomorphic function in $\zeta$ on a neighborhood of e. Since $\log K_{\mathscr{D}}(z, \zeta)=\log \eta(\Phi(z, \zeta))$ by (4.2), $\log \eta$ is holomorphic at $\Phi(z, \mathrm{e}) \in \Omega+i V$. By the chain rule, we observe for $\left(u^{\prime}, w^{\prime}\right) \in U \times W$ that

$$
\begin{aligned}
\left.\bar{\partial}_{\left(u^{\prime}, w^{\prime}\right)} \log K_{\mathscr{D}}(z, \zeta)\right|_{\zeta=\mathrm{e}} & =\left.\bar{\partial}_{\left(u^{\prime}, w^{\prime}\right)} \log \eta(\Phi(z, \zeta))\right|_{\zeta=\mathrm{e}} \\
& =\left.\left.\partial_{\bar{\partial}_{\left(u^{\prime}, w^{\prime}\right)}} \Phi(z, \zeta)\right|_{\zeta=\mathrm{e}} \log \eta(\xi)\right|_{\xi=\Phi(z, \mathrm{e})} .
\end{aligned}
$$

By (4.3) and (4.9) the last term equals

$$
\left\langle-\left.\bar{\partial}_{\left(u^{\prime}, w^{\prime}\right)} \Phi(z, \zeta)\right|_{\zeta=\mathrm{e}}, \mathcal{I}(\Phi(z, \mathrm{e}))\right\rangle=\left\langle Q\left(u, u^{\prime}\right)-\left(w^{\prime}\right)^{*}, \mathcal{I}(\Phi(z, \mathrm{e}))\right\rangle .
$$

Since $\Phi(z, \mathrm{e})=w+E$ by (4.9), we arrive at

$$
\left.\bar{\partial}_{\left(u^{\prime}, w^{\prime}\right)} \log K_{\mathscr{D}}(z, \zeta)\right|_{\zeta=\mathrm{e}}=\left\langle Q\left(u, u^{\prime}\right)-\left(w^{\prime}\right)^{*}, \mathcal{I}(w+E)\right\rangle,
$$


whence it follows that

$$
\left.\bar{\partial}_{\left(u^{\prime}, w^{\prime}\right)} \log \frac{K_{\mathscr{D}}(z, \zeta)}{K_{\mathscr{D}}(\mathrm{e}, \zeta)}\right|_{\zeta=\mathrm{e}}=\left\langle Q\left(u, u^{\prime}\right)-\left(w^{\prime}\right)^{*}, \mathcal{I}(w+E)\right\rangle+\left\langle\left(w^{\prime}\right)^{*}, \mathcal{I}(2 E)\right\rangle .
$$

By (4.4), the right-hand side is rewritten as

$$
\left\langle Q\left(u, u^{\prime}\right), \mathcal{I}(w+E)\right\rangle+\frac{1}{2}\left\langle\left(w^{\prime}\right)^{*}, \mathcal{I}(E)-2 \mathcal{I}(w+E)\right\rangle .
$$

This together with (4.6) tells us that

$$
\left.\bar{\partial}_{\left(u^{\prime}, w^{\prime}\right)} \log \frac{K_{\mathscr{D}}(z, \zeta)}{K_{\mathscr{D}}(\mathrm{e}, \zeta)}\right|_{\zeta=\mathrm{e}}=\frac{1}{2}\left\langle\left(u^{\prime}, w^{\prime}\right), \mathcal{C}(z) \circ \theta\right\rangle .
$$

Under our identification $\mathbb{C}^{N} \simeq U \times W$, the left-hand side equals

$$
\left(\left.\operatorname{grad}_{\zeta} \log \frac{K_{\mathscr{D}}(z, \zeta)}{K_{\mathscr{D}}(\mathrm{e}, \zeta)}\right|_{\zeta=\mathrm{e}} \mid\left(u^{\prime}, w^{\prime}\right)\right)=\left(\sigma_{\mathrm{e}}^{\mathscr{D}}(z) \mid\left(u^{\prime}, w^{\prime}\right)\right),
$$

where we use $T_{\mathscr{D}}(\mathrm{e})=E_{N}$. Therefore

$$
\sigma_{\mathrm{e}}^{\mathscr{D}}(z)=\frac{1}{2} \mathcal{C}(z) \hat{.}
$$

\section{A symmetry characterization by the convexity of the representative domain}

Let $D \subset \mathbb{C}^{N}$ be a domain biholomorphic to a homogeneous bounded domain.

THEOREM 5.1. Fix any point $p \in D$. Then, the representative domain $R(D, p)$ is convex if and only if $D$ is symmetric.

The proof is as follows. The domain $D$ is biholomorphic to a homogeneous Siegel domain $\mathscr{D}$. We keep to the notation in Section 4 and suppose that $\mathscr{D}$ is defined by (4.1). Let $\mathcal{C}: \mathscr{D} \rightarrow U^{\dagger} \times W^{*}$ be the Cayley transform introduced by (4.6). While the parameterized Cayley transforms $\mathcal{C}_{\mathbf{s}}$ in [5] take their images in $U \times W$, we shall see that our Cayley transform $\mathcal{C}$ is identical with $\mathcal{C}_{2 \mathbf{d}+\mathbf{b}}$ in the terminology of [5] under an appropriate identification of $U^{\dagger} \times W^{*}$ with $U \times W$, which is a slightly different one from the previous section.

We know by [5, Section 4] or [12, Section 2.1] that

$$
\eta=\Delta_{-2 \mathbf{d}-\mathbf{b}}
$$

up to a positive constant multiple. Let $\langle\cdot \mid \cdot\rangle_{2} \mathbf{d}+\mathbf{b}$ be the non-degenerate complex bilinear form on $W$ introduced in [5, Section 3.1] and $(\cdot \mid \cdot)_{2} \mathbf{d}+\mathbf{b}$ the positive definite Hermitian inner product defined by [5, (3.2)]. For $f \in W^{*}$, we define $\widetilde{f} \in W$ by

$$
\langle w \mid \widetilde{f}\rangle_{2 \mathbf{d}+\mathbf{b}}=\langle w, f\rangle \quad(w \in W)
$$

and for $g \in U^{\dagger}$, we define $\check{g} \in U$ by

$$
(\check{g} \mid u)_{2 \mathbf{d}+\mathbf{b}}=\langle u, g\rangle \quad(u \in U) .
$$

Since $\log \eta$ is holomorphic on $\Omega+i V$, the right-hand side of (4.3) is rewritten as $-D_{w} \log \eta\left(w^{\prime}\right)$. Hence it follows from (4.3) and [5, (3.1)] that

$$
\mathcal{I}(w)^{\sim}=\mathcal{I}_{2 \mathbf{d}+\mathbf{b}}(w) \quad(w \in \Omega+i V) .
$$


Moreover we see easily from (5.1) and the definition of $\langle\cdot \mid \cdot\rangle_{2 \mathbf{d}+\mathbf{b}}$ that $\mathcal{I}(E)^{\sim}=E$. Therefore we obtain from [5, (3.5)] (see also the proof of [12, Proposition 3.3]) that

$$
\mathcal{C}_{2 \mathbf{d}+\mathbf{b}}(u, w)=\left((2\langle Q(u, \cdot), \mathcal{I}(w+E)\rangle)^{\check{\nu}},(\mathcal{I}(E)-2 \mathcal{I}(w+E))^{\sim}\right) \quad((u, w) \in \mathscr{D}),
$$

which implies the coincidence of $\mathcal{C}$ and $\mathcal{C}_{2 \mathbf{d}+\mathbf{b}}$.

If the domain $\mathscr{D}$ is symmetric, we see from [5, Section 4] that the parameter $2 \mathbf{d}+\mathbf{b}$ of the Cayley transform $\mathcal{C}_{2 \mathbf{d}+\mathbf{b}}$ satisfies $2 d_{1}+b_{1}=\cdots=2 d_{r}+b_{r}$, where $r$ stands for the rank of $\mathscr{D}$. Thus, by [5, Theorem 3.1] we have the final theorem.

THEOREM 5.2. $\mathcal{C}(\mathscr{D})$ is convex if and only if $\mathscr{D}$ is symmetric.

Let $\varphi: \mathscr{D} \stackrel{\sim}{\rightarrow} D$ be a biholomorphism. By Corollary 2.2 we have $R(D, \varphi(\mathrm{e}))=$ $L(\varphi, \mathrm{e}) R(\mathscr{D}, \mathrm{e})$. This together with (4.8) gives

$$
R(D, \varphi(\mathrm{e}))=\frac{1}{2} L(\varphi, \mathrm{e}) \mathcal{C}(\mathscr{D}) \hat{.}
$$

Finally we obtain Theorem 5.1 by Lemma 3.2 and Theorem 5.2.

Acknowledgements. The authors are grateful to Professor Takeo Ohsawa for stimulating discussions and valuable comments. They also express their sincere gratitude to Professors Takaaki Nomura, Yi-Chao Xu, and Qi-Keng Lu for their interest in our work.

\section{REFERENCES}

[1] S. Bergman. Über die Existenz von Repräsentantenbereichen in der Theorie der Abbildung durch Paare von Funktionen zweier komplexen Veränderlichen. Math. Ann. 102 (1929), 430-446.

[2] S. Bergman. The kernel function and conformal mapping, second, revised edition. Mathematical Surveys, no. V. American Mathematical Society, Providence, RI, 1970.

[3] S. G. Gindikin. Analysis in homogeneous domains. Russian Math. Surveys 19-4 (1964), 1-89.

[4] C. Kai. Symmetry characterization of quasisymmetric Siegel domains by convexity of Cayley transform images. J. Lie Theory 16 (2006), 47-56.

[5] C. Kai. A characterization of symmetric Siegel domains by convexity of Cayley transform images. Tohoku Math. J. (2) 59 (2007), 101-118.

[6] C. Kai and T. Nomura. A characterization of symmetric tube domains by convexity of Cayley transform images. Differential Geom. Appl. 23 (2005), 38-54.

[7] S. Kaneyuki. On the automorphism groups of homogeneous bounded domains. J. Fac. Sci. Univ. Tokyo Sect. I 14 (1967), 89-130.

[8] A. Korányi and J. A. Wolf. Realization of hermitian symmetric spaces as generalized half-planes. Ann. of Math. (2) 81 (1965), 265-288.

[9] Q.-K. Lu. On Kaehler manifolds with constant curvature. Chinese Math.-Acta 8 (1966), 283-298.

[10] Q.-K. Lu. On the representative domain. Several complex variables (Hangzhou, 1981). Birkhäuser, Boston, MA, 1984, pp. 199-211.

[11] M. Maschler. Minimal domains and their Bergman kernel function. Pacific J. Math. 6 (1956), 501-516.

[12] T. Nomura. On Penney's Cayley transform of a homogeneous Siegel domain. J. Lie Theory 11 (2001), 185-206.

[13] T. Nomura. A characterization of symmetric Siegel domains through a Cayley transform. Transform. Groups 6 (2001), 227-260.

[14] T. Nomura. Berezin transforms and Laplace-Beltrami operators on homogeneous Siegel domains. Differential Geom. Appl. 15 (2001), 91-106.

[15] T. Nomura. Family of Cayley transforms of a homogeneous Siegel domain parametrized by admissible linear forms. Differential Geom. Appl. 18 (2003), 55-78.

[16] T. Nomura. Geometric norm equality related to the harmonicity of the Poisson kernel for homogeneous Siegel domains. J. Funct. Anal. 198 (2003), 229-267. 
[17] R. Penney. The Harish-Chandra realization for non-symmetric domains in $\mathbb{C}^{n}$. Progress in Nonlinear Differential Equations and their Applications. Vol. 20. Topics in Geometry. Birkhäuser, Boston, MA, 1996, pp. 295-313.

[18] I. I. Pyatetskii-Shapiro. Automorphic functions and the geometry of classical domains. Gordon and Breach, New York, 1969.

[19] I. Satake. Algebraic structures of symmetric domains. Iwanami Shoten, Tokyo, and Princeton University Press, Princeton, NJ, 1980.

[20] È. B. Vinberg, S. G. Gindikin and I. I. Pjateckiǔ-Šapiro. Classification and canonical realization of complex homogeneous bounded domains. Trudy Moskov. Mat. Obšč. 12 (1963), 359-388; Trans. Moscow Math. Soc. 12 (1963), 404-437.

[21] Y.-C. Xu. On the homogeneous bounded domains. Sci. Sinica Ser. A 26 (1983), 25-34.

Hideyuki Ishi

Graduate School of Mathematics

Nagoya University

Furo-cho

Chikusa-ku

Nagoya 464-8602

Japan

(E-mail: hideyuki@math.nagoya-u.ac.jp)

\section{Chifune Kai}

Faculty of Mathematics and Physics

Institute of Science and Engineering

Kanazawa University

Kakuma-machi

Kanazawa 920-1192

Japan

(E-mail:kai@staff.kanazawa-u.ac.jp) 